\title{
A unique UV flare in the optical light curve of the quasar J004457.9+412344
}

\author{
H. Meusinger ${ }^{1, a}$, M. Henze ${ }^{1,2}$, K. Birkle ${ }^{3}$, W. Pietsch², B. Williams ${ }^{4}$, \\ D. Hatzidimitriou ${ }^{5}$, R. Nesci ${ }^{6}$, S. Ertel ${ }^{1,7}$, A. Hinze ${ }^{1,8}$, T. Bertold ${ }^{9}$ and B. Kaminsky ${ }^{1,10}$ \\ ${ }^{1}$ Thüringer Landessternwarte Tautenburg, Germany \\ ${ }^{2}$ Max-Planck-Institut für extraterrestrische Physik Garching, Germany \\ ${ }^{3}$ Max-Planck-Institut für Astronomie Heidelberg, Germany \\ ${ }^{4}$ University of Washington, Seattle, USA \\ ${ }^{5}$ University of Athens, Greece \\ ${ }^{6}$ University of Roma La Sapienza, Italy \\ ${ }^{7}$ Christian-Albrechts-Universität Kiel, Germany \\ ${ }^{8}$ University Bern, Switzerland \\ ${ }^{9}$ Sternwarte Sonneberg, Germany \\ ${ }^{10}$ University Leipzig, Germany
}

\begin{abstract}
We found that the nova candidate J004457.9+412344 is a radio-quiet quasar at $z \sim 2$. Its optical long-term light curve, covering more than half a century, shows quasar typical flux variations superimposed by a spectacular single flare lasting more than one year (observer frame). We could not find comparable light curves among the several thousand catalogued radio-quiet quasars in the stripe 82 of the Sloan Digital Sky Survey. The decreasing part of the flare light curve roughly follows a power law $t^{-5 / 3}$. The quasar spectrum, the total energy of the flare, and the decline of the light curve are consistent with the tidal disruption of a $\sim 10 M_{\odot}$ giant star by a supermassive black hole of a few $10^{8} M_{\odot}$. We argue that the alternative explanation by gravitational microlensing is less likely, though it cannot be definitely excluded.
\end{abstract}

\section{INTRODUCTION}

J004457.9+412344 was originally discovered as a variable star-like source in our neighbour galaxy M 31 [1] and was classified as a nova with an unusual light curve [2] and a possible X-ray counterpart [3]. We found that the source is a faint radio-quiet type 1 quasar at $z=2.109$ seen through the outer disk of M31 [4]. An AGN misclassified as a variable star is neither unexpected nor unprecedented. However, the confusion of a luminous radio-quiet high- $z$ quasar with a nova indicates a remarkably strong outburst in the far UV that points towards a spectacular event.

\section{THE OPTICAL LONG-TERM LIGHT CURVE}

We constructed a long-term light curve based on $\sim 400 \mathrm{ob}-$ servations from the Tautenburg Schmidt telescope between 1961 and 2012 in combination with archival observations, published data, and targeted new observations from altogether 14 wide-field telescopes. J004457.9+412344 was detected at 226 epochs between 1948 and 2012. In addition, we derived useful upper limits for 12 epochs between 1900 and 1949. The light curve (Fig. 1a) clearly displays two stages, a rather quiet "ground state" at $B \sim$ 20.5 with typical fluctuations of 20 per cent and a single strong flare in 1992 that corresponds to an increase of the far UV flux by a factor of 20 . The comparison with the light curves of 8744 quasars from the stripe S82 [5,6] of the Sloan Digital Sky Survey clearly indicates that such strong+single events are extremely rare (Fig. 1b).

a e-mail:meus@tls-tautenburg.de

\section{INTERPRETATION OF THE FLARE: MICROLENSING?}

The quasar is seen through the outer disk of our neighbour galaxy at a galactocentric distance of $16 \mathrm{kpc}$ in the midplane of M 31. The optical depth, i.e., the probability for the quasar to fall into the Einstein radius of a foreground star, is $\tau=2 \cdot 10^{-4}$ corresponding to an event rate of $\sim 1$ per century for background quasars with $B<20$. The best fit of the flare light curve is achieved for a microlensing low-mass binary star with $0.3 m_{\odot}$ and $0.1 m_{\odot}$ crossing the line of sight at minimum angular distances $u_{\min }=0.05$ and 0.8 (in units of the Einstein angle), respectively. However, the probability for a magnification as strong as in J004457.9+412344 (i.e., with such a small $\left.u_{\min }\right)$ is very low. Moreover, the quasar has changed its colour in the flare by $\Delta(B-R) \sim 0.5 \mathrm{mag}$. Chromatic amplification is expected if the angular size $\alpha_{\mathrm{s}}$ of the source is comparable to $u_{\mathrm{min}}$. However, the standard accretion disk model yields $u_{\min } \geq 30 \alpha_{\mathrm{s}}$. Hence, the size of the source is negligible, even if quasar accretion disks are 3-4 times larger than predicted by the standard model (e.g., [7]). Microlensing is thus considered rather unlikely to explain the flare.

\section{4. ... OR A STELLAR TIDAL DISRUPTION EVENT?}

With the exception of the first stage of the onset of the flare, the observed shape of the light curve is in a good agreement with the predictions from the stellar tidal disruption (TD) theory (e.g., [8]): The profile is asymmetric with a sudden increase towards the peak 

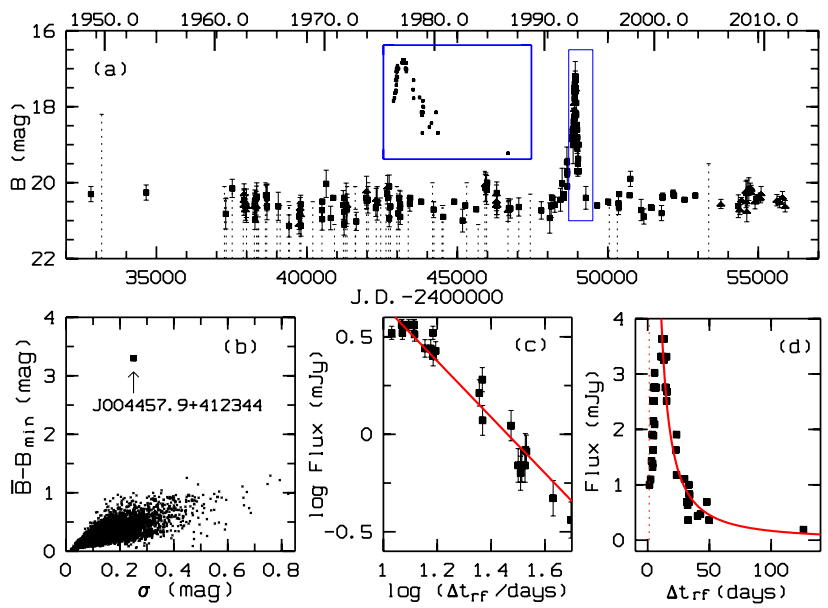

Figure 1. (a) The B band light curve of J004457.9+412344 from 1948 to 2012 (dotted lines: upper limits, insert: blow-up of the flare). (b) Maximum amplitude versus standard deviation $\sigma$ for J004457.9+412344 and 8744 quasars from the Sloan Digital Sky Survey. (c) Double-logarithmic presentation of the flare in the quasar rest-frame with linear regression of the decline. (d) As (c), but in the linear presentation (curve: $t^{-5 / 3}$ power law, vertical dotted line: onset of the flare at $t_{0}$ ).

followed by a power-law decline with an exponent of about $-5 / 3$ (Fig. 1c,d). If intrinsic and isotropic, the total radiated energy in the flare is $E_{\text {flare }}=2 \cdot 10^{54} \mathrm{erg}$. This corresponds to a mass of the disrupted star of $M_{*}=E_{\text {flare }} \eta^{-1} f_{\text {acc }}^{-1} c^{-2} \sim 10 M_{\odot}$ assuming a mass-radiation conversion efficiency $\eta \sim 0.2$ [9] and a fraction $f_{\text {acc }} \sim 0.5$ of accreted mass. The peak monochromatic UV continuum luminosity of the flare at the rest-frame wavelength $\lambda=$ $1350 \AA$ amounts to $\lambda L_{\lambda \text {,peak }} \sim 2 \cdot 10^{47} \mathrm{erg} \mathrm{s}^{-1}$. According to the TD theory, the effective temperature $T_{\text {eff }}$ of the tidal debris depends on the luminosity $L_{\text {flare }}$, the black hole mass $M_{\mathrm{bh}}$, and the radius and the mass of the star, $R_{*}, M_{*}$. From the spectrum of J004457.9+412344 and using the scaling relation for the CIV line [10] we derive $M_{\mathrm{bh}}=5 \pm 410^{8} M_{\odot}$. With $L_{\text {flare }}$ and $M_{\text {bh }}$ given and using $T_{\text {eff }}$ as a free parameter, we compute the $R_{*}-M_{*}$ relation predicted from the TD model and compare it with stellar models (Fig. 2a). While main sequence stars are clearly excluded, there exists a solution for $\sim 10 M_{\odot}$ giants where the tidal radius $R_{\mathrm{t}}$ is clearly out of the Schwarzschild radius $R_{\mathrm{S}}$ of the black hole (Fig. 2b). The maximum of the blackbody radiation is emitted at $\lambda_{\max } \sim 1300 \AA$ and shifted into the $\mathrm{B}$ band due to the redshift $z \sim 2$ of the quasar. Further, we derive from the light curve a short return time of the debris of $\Delta t_{0}=1.3$ days corresponding to a deep penetration of the star with $\beta \equiv R_{\mathrm{t}} / R_{\mathrm{p}} \sim 30$ (Fig. 2c) and $R_{\mathrm{p}} / R_{\mathrm{S}} \sim 1\left(R_{\mathrm{p}}\right.$ : pericentric distance). It can be speculated whether or not the smooth increase of the flux in the earliest phase of the flare may be related to the high penetration factor, perhaps in combination with effects from the massive accretion disk of the quasar.

\section{CONCLUSIONS}

The observation of objects at redshifts $z \sim 2-3$ provides the opportunity to study intrinsic far UV flares at optical
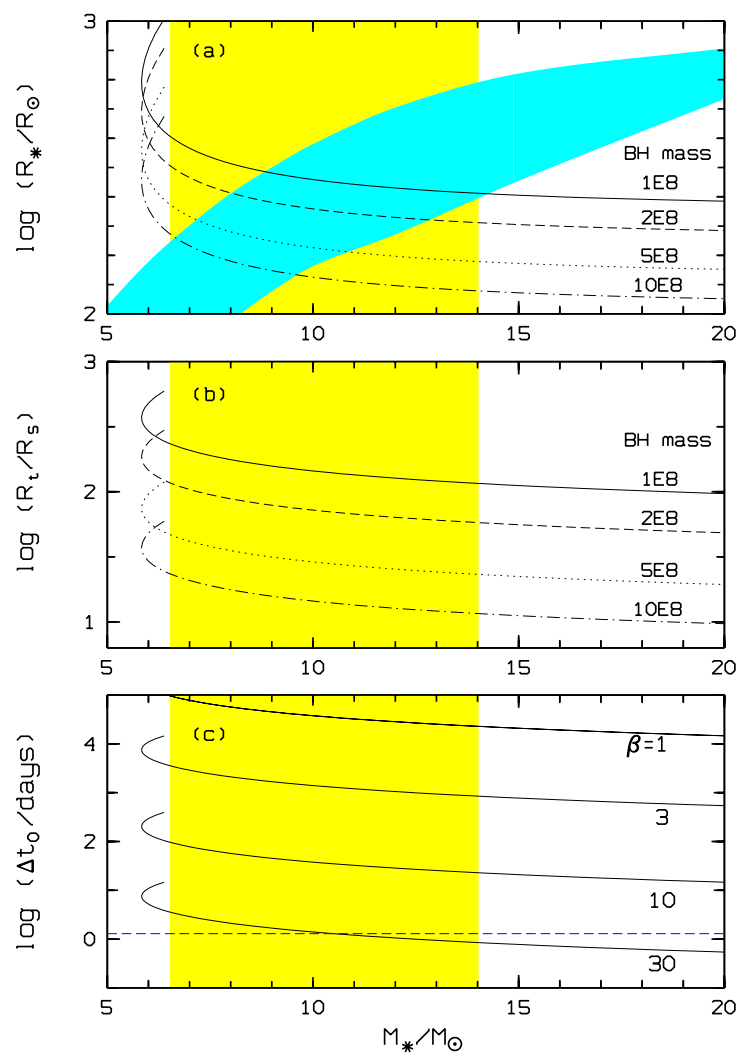

Figure 2. (a) Stellar radius $R_{*}$, (b) tidal radius $R_{\mathrm{t}}$ in units of the Schwarzschild radius $R_{\mathrm{S}}$, and $(c)$ return time $\Delta t_{0}$ as function of the mass $M_{*}$ of the tidally disrupted star for different black hole masses in $(a)$ and $(b)$ and for different penetration ratios $\beta$ in $(c)$. The shaded stripe from bottom left to top right in (a) covers the data range for giant stars from stellar models [11]. The vertical stripe in all three panels marks the possible range of stellar masses for the TD interpretation of the flare. The dashed line in $(c)$ indicates $\Delta t_{0}$ from the light curve.

wavelengths. We present a single, outstandingly strong far UV bump in the B band long-term light curve of the $z \sim 2$ quasar J004457.9+412344 which is highly unusual for radio-quiet quasars. Its properties appear broadly consistent with the tidal disruption of a $\sim 10 M_{\odot}$ giant star by a $2 . .5 \cdot 10^{8} M_{\odot}$ black hole [4]. If not caused in this way, the outburst of J004457.9+412344 would hint to a rare transient phenomenon which mimics TD flares. It can be assumed that projects like Pan-STARRS and LSST will discover more of them.

\section{References}

[1] Nedialkov, P., et al., Inf. Bull. Var. Stars 4411, (1996)

[2] Sharov, A. S., et al., Astron. Lett. 24, (1998) 445

[3] Pietsch, W., et al., A\&A 442, (2005) 879

[4] Meusinger, H., et al., A\&A 512, (2010) A1

[5] Bramich, D. M., et al., MNRAS 386, (2008) 887

[6] Meusinger, H., et al., A\&A 525, (2011) A37

[7] Morgan, C. W., et al., ApJ 712, (2010) 1129

[8] Gezari, S., this conference

[9] Davis, S. W. \& Laor, A., ApJ 728, (2011) 98

[10] Vestergaard, M. \& Peterson, B. M., ApJ 641, (2006) 689

[11] Salasnich, B., et al. A\&A 361, (2000) 1023 